\title{
Oncostatin $M$ is a potential agent for the treatment of obesity and related metabolic disorders: a study in mice
}

\author{
Tadasuke Komori ${ }^{1}$ - Minoru Tanaka ${ }^{2}$ - Hiroto Furuta ${ }^{3}$ Takashi Akamizu ${ }^{3}$. \\ Atsushi Miyajima $^{4}$ - Yoshihiro Morikawa ${ }^{1}$
}

Received: 3 March 2015 / Accepted: 13 April 2015 / Published online: 14 May 2015

(C) Springer-Verlag Berlin Heidelberg 2015

\begin{abstract}
Aims/hypothesis Obesity and insulin resistance are closely associated with adipose tissue dysfunction caused by the abnormal recruitment of inflammatory cells, including macrophages. Oncostatin M (OSM), a member of the IL-6 family of cytokines, plays important roles in a variety of biological functions including the regulation of inflammatory responses. In previous reports, we have demonstrated that mice deficient in the OSM receptor $\beta$ subunit show obesity, adipose tissue inflammation, insulin resistance and hepatic steatosis, all of which are exacerbated by feeding the mice a high-fat diet. These results prompted us to test the therapeutic effects of OSM on obesity-induced metabolic disorders using mouse models of obesity.

Methods In diet-induced obese and $o b / o b$ mice, metabolic variables were assessed physiologically, histologically and biochemically after the intraperitoneal injection of recombinant mouse OSM twice a day for 1 week.
\end{abstract}

Electronic supplementary material The online version of this article (doi:10.1007/s00125-015-3613-9) contains peer-reviewed but unedited supplementary material, which is available to authorised users.

Yoshihiro Morikawa

yoshim@wakayama-med.ac.jp

1 Department of Anatomy and Neurobiology, Wakayama Medical University, 811-1 Kimiidera, Wakayama 641-8509, Japan

2 Department of Regenerative Medicine, Research Institute, National Center for Global Health and Medicine, Tokyo, Japan

3 The First Department of Medicine, Wakayama Medical University, Wakayama, Japan

4 Laboratory of Cell Growth and Differentiation, Institute of Molecular and Cellular Biosciences, The University of Tokyo, Tokyo, Japan
Results Treatment with OSM improved obesity, adipose tissue inflammation, insulin resistance and hepatic steatosis in both mouse models. Although OSM reduced food intake, such therapeutic effects of OSM were observed even under pair-feeding conditions. Functionally, OSM directly changed the phenotype of adipose tissue macrophages from M1 type (inflammatory) to M2 type (anti-inflammatory). In the liver, OSM suppressed the expression of genes related to fatty acid synthesis and increased the expression of genes related to fatty acid oxidation. Furthermore, OSM decreased lipid absorption and increased the expression of active glucagon-like peptide-1 in the intestine.

Conclusions/interpretation We showed that OSM is a novel candidate to act as a powerful therapeutic agent for the treatment of obesity-induced metabolic disorders.

Keywords Adipose tissue inflammation · Hepatic steatosis · High-fat diet · Insulin resistance $\cdot$ Lipid absorption · Metabolic disorders $\cdot$ Obesity $\cdot$ Therapeutic agent

$\begin{array}{ll}\text { Abbreviations } \\ \text { ACSL } & \text { Long-chain acyl-CoA synthetase } \\ \text { ATM } & \text { Adipose tissue macrophage } \\ \text { BW } & \text { Body weight } \\ \text { DIO } & \text { Diet-induced obese } \\ \text { ERK } & \text { Extracellular signal-regulated kinase } \\ \text { FGF21 } & \text { Fibroblast growth factor } 21 \\ \text { GLP-1 } & \text { Glucagon-like peptide-1 } \\ \text { HFD } & \text { High-fat diet } \\ \text { ipGTT } & \text { Intraperitoneal glucose tolerance test } \\ \text { ITT } & \text { Insulin tolerance test } \\ \text { OSM } & \text { Oncostatin M } \\ \text { OSMR } \beta & \text { OSM receptor } \beta \text { subunit } \\ \text { RIPA } & \text { Radioimmune precipitation assay }\end{array}$


SAA Serum amyloid A

STAT Signal transducer and activator of transcription

SVF Stromal vascular fraction

\section{Introduction}

There is increasing evidence that obesity induces the development of insulin resistance and type 2 diabetes via underlying chronic low-grade inflammation in adipose tissue [1-3]. However, the mechanisms of obesity-related metabolic diseases are not fully understood. In addition, obesity leads to the ectopic accumulation of lipids in various tissues, including liver, skeletal muscle and heart $[4,5]$. Especially, accumulation of lipids in hepatocytes induces inflammatory changes in the liver, called non-alcoholic steatohepatitis, which is a risk factor for the development of insulin resistance, liver cirrhosis and hepatocellular carcinoma $[6,7]$. Therefore, a therapeutic approach to obesity-related metabolic diseases is required not only for obesity but also for insulin resistance and hepatic steatosis.

Oncostatin M (OSM) belongs to the IL-6 family of cytokines and exhibits a variety of biological effects on target cells through its binding to a heterodimeric receptor complex comprising the OSM receptor $\beta$ subunit (OSMR $\beta$ ) and the common receptor subunit of IL-6 family, gp130 [8-10]. It has been reported that OSM is produced primarily by various inflammatory cells, including neutrophils, eosinophils and macrophages [11-13], and plays an important role in the modulation of inflammatory responses [8, 13]. However, little has been reported on the involvement of OSM in metabolic disorders.

In a previous study, we demonstrated that OSM induces the anti-inflammatory (M2) phenotype of adipose tissue macrophages (ATMs) and that disruption of the $O s m r \beta$ gene (also known as Osmr) results in adipose tissue inflammation and insulin resistance with mature-onset obesity through the phenotypic change of ATMs to the inflammatory type (M1) under normal diet conditions [14]. In addition, obesity and related metabolic diseases, including hepatic steatosis, were exacerbated in mice deficient in OSMR $\beta$ under high-fat diet (HFD) conditions [15]. These findings suggest that OSM has beneficial effects on the development of obesity, adipose tissue inflammation, insulin resistance and hepatic steatosis. In the present study, to verify OSM as a candidate for novel therapeutic agents in the metabolic syndrome, we examined the effects of OSM on the metabolic symptoms and variables in both dietinduced obese (DIO) mice and genetically obese $(o b / o b)$ mice.

\section{Methods}

Animals Male C57BL/6J mice were purchased from Nihon SLC (Hamamatsu, Japan). Male $o b / o b$ mice were obtained from our breeding colony using heterozygous $(o b /+)$ breeding pairs. All mice were housed in specific pathogen-free facilities under light ( $12 \mathrm{~h}$ light-dark cycle)-, temperature $\left(22-25^{\circ} \mathrm{C}\right)$ and humidity (50-60\% relative humidity)-controlled conditions, with free access to food and water. Until 8 weeks of age, all mice were fed a normal diet consisting of $13.3 \%$ of energy content from fat (MF; Oriental Yeast, Tokyo, Japan). At all times, the experiments were performed under the control of the Animal Research Control Committee in accordance with the Guidelines for Animal Experiments of Wakayama Medical University, the Japanese Government Notification on Feeding and Safekeeping of Animals (No. 6), and the National Institutes of Health Guide for the Care and Use of Laboratory Animals (NIH publication No.80-23, revised 1978). All efforts were made to minimise the number of animals used and their suffering.

Treatment with OSM Starting at 8 weeks of age, male C57BL/6J mice were placed on an HFD consisting of 56.7\% of the energy content from fat (High Fat Diet 32; CLEA Japan, Tokyo, Japan) and were fed in individual cages for 8 weeks. Male $o b / o b$ mice were fed with normal chow (MF) in individual cages. These DIO mice and $o b / o b$ mice were treated intraperitoneally with vehicle or recombinant mouse OSM (R \& D Systems, Minneapolis, MN, USA) at the indicated dose (1.25, 3.75 and $12.5 \mathrm{ng} / \mathrm{g}$ body weight [BW]) twice a day (at 10:00 and 18:00 hours) for 1 week. In the pair-feeding experiments, paired-fed mice were given vehicle and the same amount of food as that consumed by OSM-treated mice. To investigate the direct effects of OSM on the liver, DIO mice were deeply anaesthetised with isoflurane and administered intraportally with vehicle or OSM (12.5 ng/g BW) as described previously [15].

Measurement of body temperature, locomotor activity and $\mathrm{O}_{2}$ consumption See electronic supplementary material (ESM) Methods for details.

Isolation of ATMs and flow cytometry Stromal vascular fraction (SVF) isolation and flow cytometry were performed as described previously $[14,15]$. The primary antibodies used in the flow cytometry are shown in ESM Table 1. For the isolation of ATMs, the cells in the SVF were incubated with anti-CD16/CD32 antibodies (BD Biosciences, San Jose, CA, USA), followed by incubation with FITC-conjugated anti-F4/ 80 antibody. F4/80-stained cells in the SVF were obtained by using a magnet-activated cell-sorting (MACS) anti-FITC Multisort kit (Miltenyi Biotech, Aubum, CA, USA) and an autoMACS Pro Separator (Miltenyi Biotech). Sorted ATMs were cultured in DMEM (Invitrogen, Carlsbad, CA, USA) with $10 \%$ (wt/vol.) FCS, $100 \mathrm{U} / \mathrm{ml}$ penicillin (Invitrogen) and $100 \mu \mathrm{g} / \mathrm{ml}$ streptomycin (Invitrogen) for $2 \mathrm{~h}$. The cells were then treated with vehicle or $50 \mathrm{ng} / \mathrm{ml}$ of OSM and 
maintained for 1 or $2 \mathrm{~h}$. All cells were cultured at $37^{\circ} \mathrm{C}$ in a humidified atmosphere of $5 \% \mathrm{CO}_{2}$.

Western blot analysis Western blot analysis was performed as previously described [14, 15]. Tissue lysates were prepared using radioimmune precipitation assay (RIPA) buffer (Upstate Biotechnology, Lake Placid, NY, USA). The primary antibodies used in the present study are shown in ESM Table 1.

Immunohistochemistry Immunofluorescence staining in the hypothalamus and liver was performed as previously described [15]. The mice were deeply anaesthetised with isoflurane and transcardially perfused with ice-cold $0.85 \%$ (wt/vol.) $\mathrm{NaCl}$ followed by ice-cold Zamboni's fixative $(2 \%$ [wt/vol.] PFA and $0.2 \%$ [wt/vol.] picric acid in $0.1 \mathrm{~mol} / \mathrm{PBS}$ ). The primary antibodies used in the present study are shown in ESM Table 1.

Analysis of glucose metabolism Blood glucose levels were measured with a glucose measurement device (Glucocard GT1640; Arkray, Kyoto, Japan). The serum insulin concentration was determined using kits from Morinaga (Tokyo, Japan). An intraperitoneal glucose tolerance test (ipGTT) and insulin tolerance test (ITT) were performed as described previously [14, 15]. For the ipGTT, the mice were fasted for $16 \mathrm{~h}$ and received an intraperitoneal injection of D-glucose ( $1 \mathrm{~g} / \mathrm{kg} \mathrm{BW}$ for DIO mice and $0.5 \mathrm{~g} / \mathrm{kg} \mathrm{BW}$ for $o b / o b$ mice). For the ITT, mice were fasted for $4 \mathrm{~h}$ and received an intraperitoneal injection of human insulin (1 U/kg BW for DIO mice and $5 \mathrm{U} / \mathrm{kg}$ BW for $o b / o b$ mice).

ELISA Serum concentrations of TNF- $\alpha$, IL-10, adiponectin, leptin and serum amyloid A (SAA) were measured with respective ELISA kits from R \& D Systems, Morinaga and Invitrogen as described previously $[14,15]$. For the measurement of active glucagon-like peptide-1 (GLP-1) levels in the intestine, tissue samples were homogenised in RIPA buffer with dipeptidyl peptidase-4 inhibitor at a dose of $50 \mu \mathrm{mol} / \mathrm{l}$ (Millipore, Billerica, MA, USA). The concentration of active GLP-1 in the intestine was measured with an ELISA kit (Millipore).

Analysis of lipid metabolism Measurement of lipid content in the serum and liver was performed as described previously $[14,15]$. Mice were fasted for $4 \mathrm{~h}$ to remove the effects of food intake on lipid metabolism. The serum levels of triacylglycerols, total cholesterol and NEFA were measured at Nagahama Life Science Laboratory (Nagahama, Japan). The serum concentration of $\beta$-hydroxybutyrate was measured with the EnzyChrom ketone body assay kit (Bioassay Systems, Hayward, CA, USA). The content of triacylglycerols and total cholesterol in the liver was analysed at Skylight Biotech (Akita, Japan). Measurement of lipid contents in the faeces was performed by modified Kang's methods [16]. Faeces were collected for 2 days from mice in cages with lifted-wire bottoms. The faeces were then dried at $60^{\circ} \mathrm{C}$ for $1 \mathrm{~h}$ and the dry weight was determined. Total lipids were extracted in chloroform-methanol (2:1 vol./vol.) and evaporated to dryness. The total amount of lipids was measured by subtracting the weight of a tube without lipids from the weight of the same tube containing lipids. Then the dried lipids were resuspended in 5\% (wt/vol.) Triton X-100. The amount of triacylglycerols in the extract was measured using the EnzyChrom triglyceride assay kit (Bioassay Systems). The percentage of lipid absorption was calculated as ([lipid consumed]-[faecal lipid]) / (lipid consumed) $\times 100$.

Quantitative real-time PCR Quantitative real-time PCR was performed with some modifications as described previously [14, 15]. Total RNAs were prepared using TRI reagent (Molecular Research Center, Cincinnati, OH, USA). The TaqMan Gene Expression Assays (Applied Biosystems, Foster City, CA, USA) used in the present study are shown in ESM Table 2.

Statistical analysis The results are shown as means \pm SEM. Comparisons between two groups were analysed by Student's $t$ test. For multiple-group comparisons, ANOVA followed by the post hoc Bonferroni test was used. The criterion for statistical significance was $p<0.05$.

\section{Results}

OSM treatment improves obesity, glucose intolerance and insulin resistance in obese mice Initially, we examined the dose-response effects of OSM on obesity, glucose intolerance and insulin resistance in DIO mice. BW, food intake, glucose intolerance and insulin resistance were significantly reduced after injection of OSM at $12.5 \mathrm{ng} / \mathrm{g} \mathrm{BW}$ (Fig. 1). When injected at $3.75 \mathrm{ng} / \mathrm{g} \mathrm{BW}$, OSM did not reduce BW or food intake (Fig. 1a, c), whereas glucose intolerance and insulin resistance was improved (Fig. 1d-g). None of these metabolic variables were changed in DIO mice injected with OSM at a dose of $1.25 \mathrm{ng} / \mathrm{g} \mathrm{BW}$ (Fig. 1).

Compared with vehicle-treated mice, the BW, adipose tissue weight and serum leptin levels were decreased in the Vehicle(PF) group (vehicle-treated mice pair-fed with OSMtreated mice) as well as in the OSM-treated mice (Fig. 2a-c, ESM Fig. 1a-c, Table 1). On the other hand, the liver weight was reduced only in the OSM group (Fig. 2c, ESM Fig. 1c). The serum adiponectin levels did not differ between the three groups (Table 1; ESM Table 3) and the blood glucose and serum insulin levels in the Vehicle(PF) group were almost the same as in the vehicle-treated group (Table 1; ESM Table 3). Although glucose intolerance and insulin resistance 
a
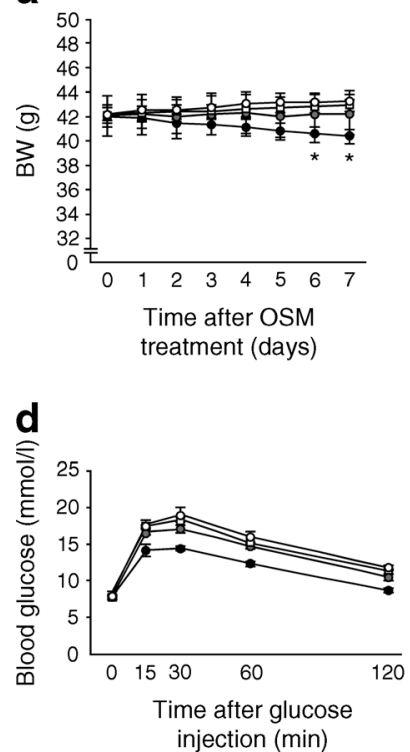

Fig. 1 Dose-response effects of OSM on BW and glucose metabolism in DIO mice. DIO mice were treated with OSM $(1.25,3.75$ and $12.5 \mathrm{ng} / \mathrm{g}$ BW) twice a day for 1 week. (a-c) BW (a), tissue weight (b) and food intake (c) in the vehicle-treated and OSM-treated groups $(n=6)$. EWAT, epididymal white adipose tissue; SWAT, subcutaneous white adipose tissue.

were improved in the OSM-treated group, they were not improved in the Vehicle(PF) group (Fig. 2d-i).

\section{OSM treatment ameliorates adipose tissue inflammation} in obese mice The serum TNF- $\alpha$ and SAA levels were lower in the OSM-treated group than in the vehicle-treated group, whereas the serum IL-10 level was higher in the OSM group in both mouse models of obesity (Table 1; ESM Table 3). In the adipose tissue, the percentage and total number of F4/80positive macrophages were lower in the SVF of the OSMtreated group compared with the vehicle-treated group (Fig. 3a-c; ESM Fig. 2a-c). The percentage of CD11cpositive M1 ATMs was lower in the OSM-treated group than in the vehicle-treated group, while the OSM group exhibited a higher percentage of CD206-positive M2 ATMs (Fig. 3d, e; ESM Fig. 2d, e). Furthermore, the ratio of M1 to M2 (CD11cpositive / CD206-positive) ATMs was decreased in the OSMtreated group compared with the vehicle-treated group (Fig. 3f; ESM Fig. 2f).

In the adipose tissue and SVF, the expression levels of genes encoding inflammatory markers, Tnf- $\alpha$ (also known as Tnf), Il-1 $\beta$ (Illb), Ifn- $\gamma$ (Ifng), Ccr 2 (encoding C-C chemokine receptor 2), Mcp-1 (Ccl2, encoding monocyte chemoattractant protein-1) and Tlr4 (encoding toll-like receptor 4), were lower in the OSM-treated group than in the vehicle-treated group, whereas there was no difference in the expression of $\mathrm{Il}-6$ (Il6) (Fig. 3g, h; ESM Fig. 2g, h). In addition, the expression levels of genes encoding anti-inflammatory markers, $\mathrm{Il}-10$ (Illo), $\mathrm{Il}$ -
C

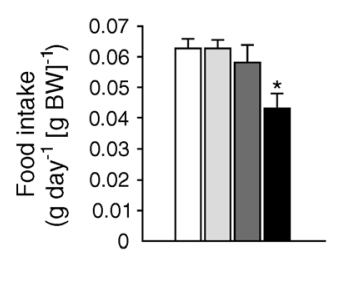

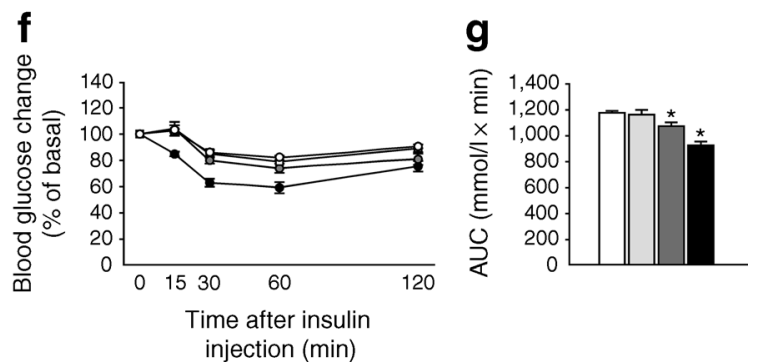

(d-g) Blood glucose levels during the ipGTTs (d) and ITTs (f) $(n=6-9)$ and AUCs for blood glucose in the ipGTTs (e) and ITTs (g). White circles and bars, Vehicle; white squares and light grey bars, OSM $1.25 \mathrm{ng} / \mathrm{g} \mathrm{BW}$; dark grey circles and bars, OSM $3.75 \mathrm{ng} / \mathrm{g} \mathrm{BW}$; black circles and bars, OSM $12.5 \mathrm{ng} / \mathrm{g}$ BW. Data are means \pm SEM. ${ }^{*} p<0.05$ compared with Vehicle

13 (Il13), Mgl1 (Clec10a, encoding macrophage galactosetype C-type lectin 1), Mgl2 and Adipoq, were higher in the OSM-treated group than in the vehicle-treated group (Fig. 3g, h; ESM Fig. 2g, h). Such changes in the OSM group were not observed in the Vehicle(PF) group (Fig. 3a-h; ESM Fig. 2ah). To investigate the direct effects of OSM on ATMs, we isolated ATMs from the epididymal white adipose tissue of DIO mice. OSM increased the expression of antiinflammatory marker genes $\mathrm{Il}-10, \mathrm{Mgll}$, and $\mathrm{Mgl2}$, in cultured ATMs within $2 \mathrm{~h}$ after the treatment (Fig. 3i). However, OSM had no effect on the gene expression of inflammatory cytokines, including Tnf- $\alpha$ and $I l-1 \beta$ (Fig. 3i).

OSM treatment alleviates hepatic steatosis in obese mice There were fewer Oil Red O-positive lipid droplets in the liver of OSM-treated mice than in the liver of vehicletreated mice, whereas the glycogen content was increased in the OSM group (Fig. 4a; ESM Fig. 3a). In addition, the levels of total cholesterol and triacylglycerols in the liver (Fig. 4b, c; ESM Fig. 3b, c), as well as the serum concentrations of total cholesterol, triacylglycerols and NEFA (Table 1; ESM Table 3), were decreased in the OSM-treated group compared with the vehicle-treated group. The serum concentration of $\beta$ hydroxybutyrate in the OSM-treated group was similar to that observed in the vehicle-treated group (Table 1). The expression levels of genes related to fatty acid synthesis, fatty acid synthase (Fas), steroyl CoA desaturase-1 $(S c d-1, S c d 1)$ and sterol regulatory-element binding transcription factor-1 
a

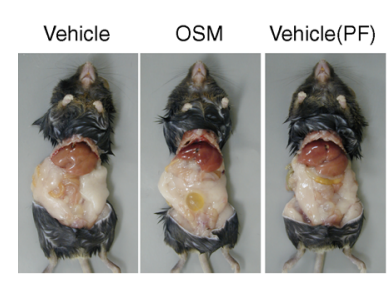

b

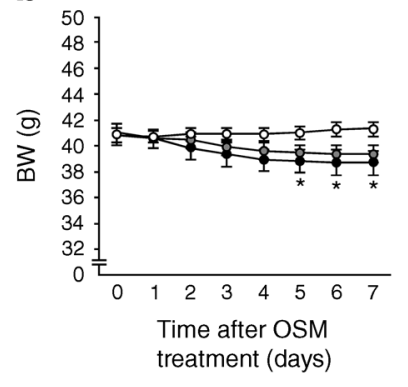

C

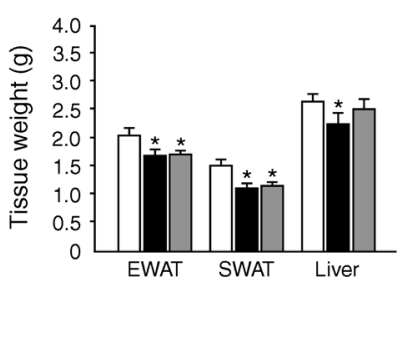

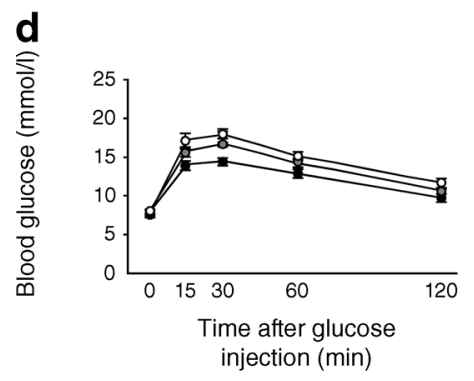
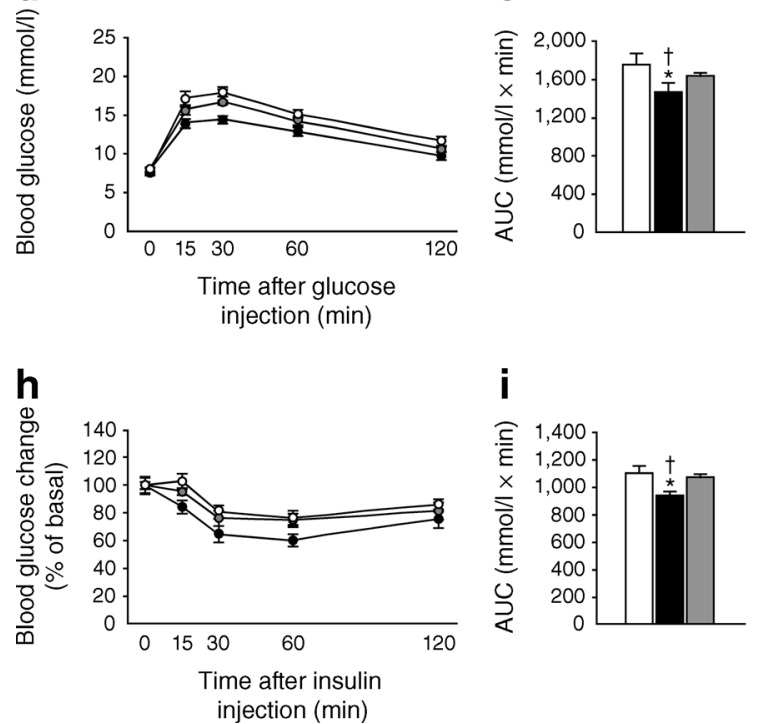

e

f

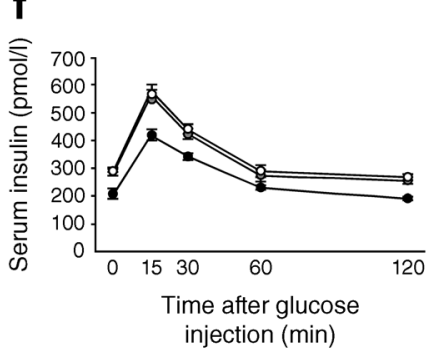

i

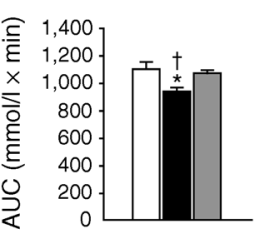

Fig. 2 Effect of OSM-induced hypophagia on BW and glucose metabolism in DIO mice. DIO mice were treated with OSM (12.5 ng/g BW) twice a day for 1 week. (a-c) Representative images (a), BW (b) and tissue weights (c) in the vehicle-treated, OSM-treated and Vehicle(PF) groups $(n=6)$. EWAT, epididymal white adipose tissue; SWAT, subcutaneous white adipose tissue. $(\mathbf{d}-\mathbf{i})$ Blood glucose $(\mathbf{d})$ and serum insulin levels (f) during the ipGTTs $(n=6-9)$ and blood glucose levels during the

(Srebf-1, Srebf1), were decreased in the liver of the OSMtreated group (Fig. 4d; ESM Fig. 3d). These changes seen in the OSM-treated group were not observed in the Vehicle(PF) group (Fig. 4; ESM Fig. 3).

OSMR $\beta$ was expressed in albumin-positive hepatocytes in the liver of DIO mice (Fig. 5a). Intraportal injection of OSM resulted in phosphorylation of signal transducer and activator of transcription 3 (STAT3) and extracellular signal-regulated kinase (ERK) in the liver (Fig. 5b), especially in the OSMR $\beta$ positive hepatocytes (Fig. $5 \mathrm{c}$ ). In addition, the intraportal injection of OSM decreased the expression of Fas, a gene related to fatty acid synthesis, and increased the expression of Acsl3 (encoding long-chain acyl-CoA synthetase 3, ACSL3) and Acsl5, related to fatty acid oxidation (Fig. 5d). However, the intraportal injection of OSM had no effect on the expression of other genes related to fatty acid oxidation, Acox 1 (encoding acyl-CoA oxidase 1), Cptla (encoding carnitine palmitoyltransferase 1a) and Ucp2 (encoding uncoupling

ITTs $(\mathbf{h})(n=6-9)$. AUCs for blood glucose $(\mathbf{e}, \mathbf{i})$ and serum insulin $(\mathbf{g})$ in the ipGTTs (e, g) and ITTs (i) are shown. White circles and bars, Vehicle; black circles and bars, OSM; grey circles and bars, Vehicle(PF). Data are means \pm SEM. ${ }^{*} p<0.05$ compared with Vehicle; ${ }^{\dagger} p<0.05$ compared with Vehicle(PF); in (b) $* p<0.05$ for both OSM and Vehicle(PF) compared with Vehicle

protein 2) or on genes related to fatty acid transport, $C d 36$ and Fabp4 (encoding fatty acid binding protein 4) (Fig. 5d).

OSM treatment decreases lipid absorption in obese mice without changing the variables of energy expenditure Next, we investigated the effects of OSM on lipid absorption in the intestine of DIO mice. The total lipid and triacylglycerol contents in the faeces were increased in the OSM-treated group compared with the vehicle-treated group, although the quantity of faeces produced by the two groups was similar (Fig. 6a-c). In addition, the percentage of lipid absorption was decreased in the OSM group (Fig. 6d). However, OSM did not affect the $\mathrm{O}_{2}$ consumption, body temperature, locomotor activity or the expression of genes related to energy expenditure in the brown adipose tissue, $U c p 1$ and $P G C l \alpha$ (also known as Ppargcla, encoding peroxisome proliferatoractivated receptor- $\gamma$-coactivator- $1 \alpha$ ), and skeletal muscle, Ucp3 and Cpt1b (ESM Fig. 4). 
Table 1 Metabolic variables in the serum of OSM-treated DIO mice $(n=6-8)$

\begin{tabular}{|c|c|c|c|}
\hline Serum concentration & Vehicle & OSM & Vehicle(PF) \\
\hline Leptin (ng/ml) & $27.5 \pm 1.3$ & $16.0 \pm 2.8^{* \dagger}$ & $21.7 \pm 1.5^{*}$ \\
\hline Adiponectin $(\mu \mathrm{g} / \mathrm{ml})$ & $22.3 \pm 1.1$ & $21.6 \pm 1.0$ & $21.6 \pm 1.0$ \\
\hline SAA (ng/ml) & $27.6 \pm 2.0$ & $22.3 \pm 1.3^{* \dagger}$ & $25.7 \pm 3.1$ \\
\hline $\mathrm{TNF}-\alpha(\mathrm{pg} / \mathrm{ml})$ & $6.31 \pm 0.26$ & $5.35 \pm 0.30^{* \dagger}$ & $5.99 \pm 0.13$ \\
\hline IL-10 (pg/ml) & $16.6 \pm 1.5$ & $27.6 \pm 4.2 * *^{\dagger}$ & $17.2 \pm 1.8$ \\
\hline Glucose (fed) (mmol/l) & $11.2 \pm 0.1$ & $9.9 \pm 0.3 * \dagger$ & $11.1 \pm 0.4$ \\
\hline Insulin (fed) (pmol/l) & $995.3 \pm 104.9$ & $600.2 \pm 109.2 *^{\dagger}$ & $968.5 \pm 123.9$ \\
\hline Glucose (fasted) (mmol/l) & $8.30 \pm 0.29$ & $7.28 \pm 0.30^{* \dagger}$ & $7.90 \pm 0.39$ \\
\hline Insulin (fasted) (pmol/1) & $326.7 \pm 44.2$ & $194.9 \pm 38.7 *^{\dagger}$ & $272.1 \pm 44.2$ \\
\hline Total cholesterol (fed) (mmol/l) & $4.37 \pm 0.10$ & $3.43 \pm 0.08^{* \dagger}$ & $3.95 \pm 0.13^{*}$ \\
\hline Triacylglycerol (fed) (mmol/l) & $1.90 \pm 0.25$ & $1.23 \pm 0.08^{* \dagger}$ & $1.70 \pm 0.17$ \\
\hline NEFA (fed) (mmol/l) & $1.51 \pm 0.04$ & $1.30 \pm 0.06^{* \dagger}$ & $1.48 \pm 0.04$ \\
\hline$\beta$-Hydroxybutyrate (fed) (mmol/l) & $0.56 \pm 0.04$ & $0.48 \pm 0.02$ & $0.51 \pm 0.03$ \\
\hline
\end{tabular}

Data are means \pm SEM

In the fed state, mice were fasted for $4 \mathrm{~h}$ before the experiments to eliminate the effect of feeding on glucose and lipid metabolism. In the fasted state, mice were fasted for $16 \mathrm{~h}$ before the experiments

${ }^{*} p<0.05$ compared with Vehicle; ${ }^{\dagger} p<0.05$ compared with Vehicle(PF)

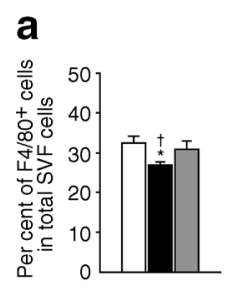

b

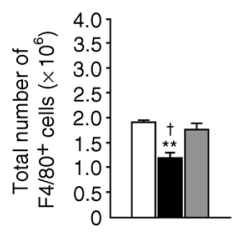

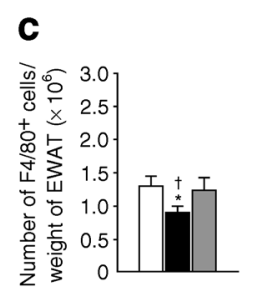
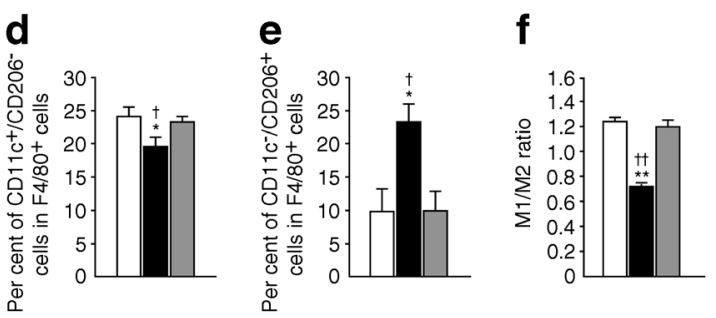
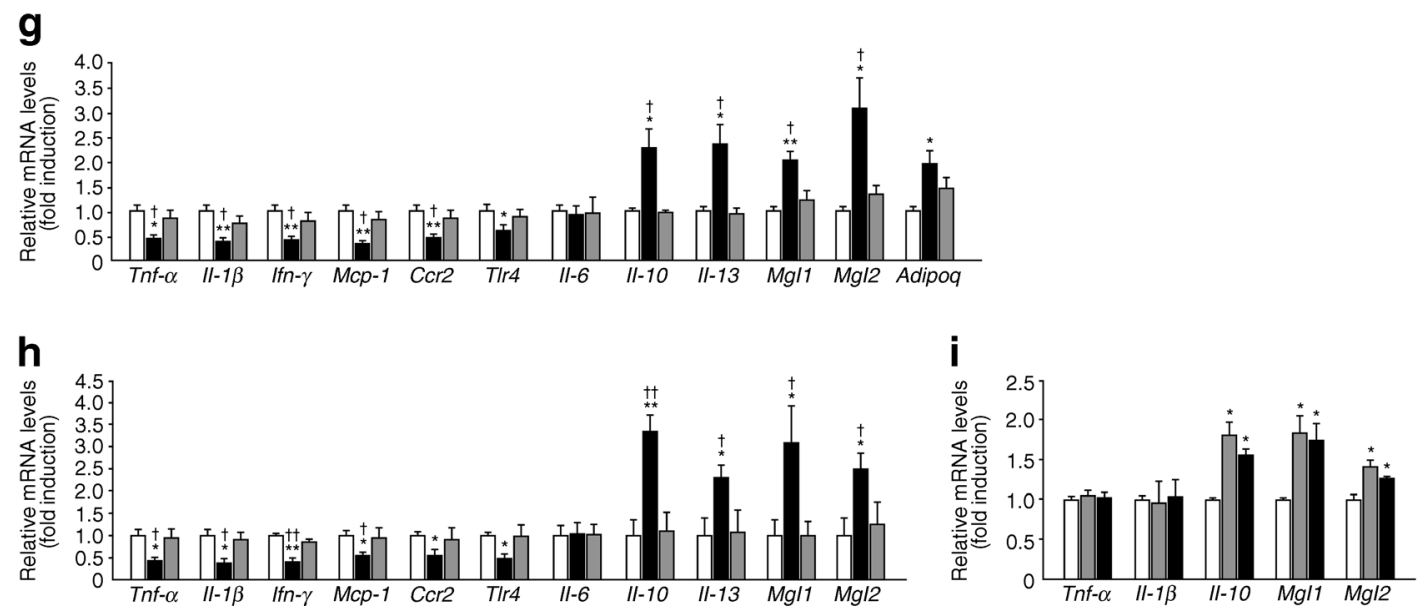

Fig. 3 Effect of OSM on adipose tissue inflammation in DIO mice. DIO mice were treated with OSM $(12.5 \mathrm{ng} / \mathrm{g} \mathrm{BW})$ twice a day for 1 week. (ac) The percentages (a) and the total number (b) of F4/80-positive cells among the total numbers of cells in the SVF of the epididymal fat pads in vehicle-treated, OSM-treated and Vehicle(PF) groups $(n=6)$. The total number of macrophages was normalised by the weights of the epididymal fat pads (c). EWAT, epidymal white adipose tissue. (d, e) The percentages of CD11 c-positive (d) and CD206-positive (e) cells in the F4/80-positive cells $(n=6)$. (f) The ratio of CD11c-positive cells to CD206-positive cells

$(n=6) .(\mathbf{g}, \mathbf{h})$ Gene expression in the adipose tissue $(\mathbf{g})$ and the SVF (h) $(n=6)$. White bars, Vehicle; black bars, OSM; grey bars, Vehicle(PF). (i) The direct effect of OSM on phenotypes of ATMs. ATMs were isolated from EWAT of DIO mice and treated with OSM $(50 \mathrm{ng} / \mathrm{ml})$ for 1 and $2 \mathrm{~h}$ $(n=4)$. The expression of genes in the ATMs is shown. White bars, Control; grey bars, OSM $1 \mathrm{~h}$; black bars, OSM 2 h. Data are means \pm SEM. ${ }^{*} p<0.05$ and ${ }^{* *} p<0.01$ compared with Vehicle (a-h) or Control (i); ${ }^{\dagger} p<0.05$ and ${ }^{\dagger \dagger} p<0.01$ compared with Vehicle(PF) (a-h) 
a

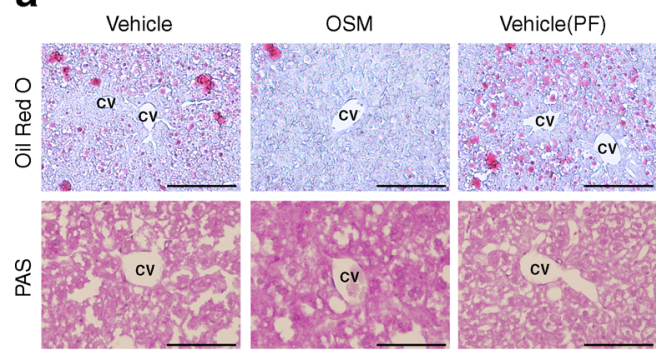

b
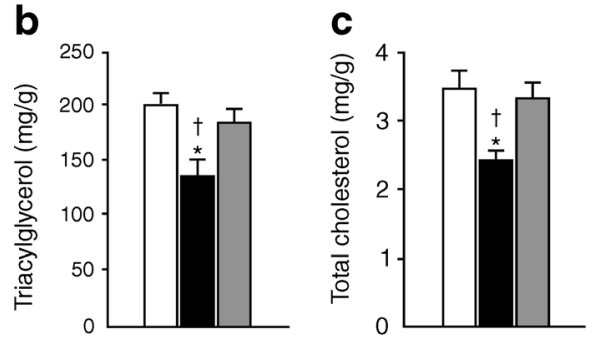

d

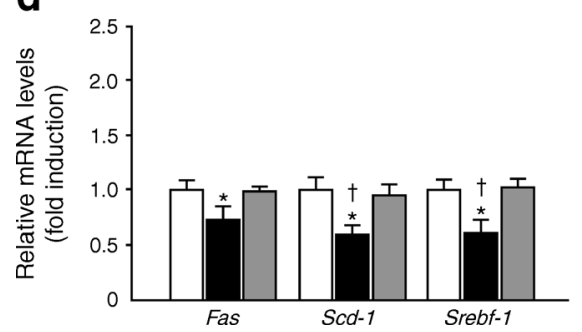

Fig. 4 Effect of OSM on liver lipid metabolism in DIO mice. DIO mice were treated with OSM (12.5 ng/g BW) twice a day for 1 week. (a) Oil Red $\mathrm{O}$ and periodic acid-Schiff (PAS) staining in the livers of the vehicletreated, OSM-treated and Vehicle(PF) groups. CV, central vein. Scale bar, $100 \mu \mathrm{m}$ (Oil Red O) or $50 \mu \mathrm{m}$ (PAS). (b-d) The content of triacylglycerols (b) and total cholesterol (c) and the expression of genes related to fatty acid synthesis (d) in the livers $(n=6)$. White bars, Vehicle; black bars, OSM; grey bars, Vehicle(PF). Data are means \pm SEM. * $p<0.05$ compared with Vehicle; ${ }^{\dagger} p<0.05$ compared with Vehicle(PF)

OSM treatment elevates the expression of GLP-1 in the intestine of obese mice Next, we examined how OSM decreased food intake in DIO mice. Expression of OSMR $\beta$ was not observed in the hypothalamus of DIO mice (ESM Fig. 5). However, the expression of the gene encoding proglucagon, the precursor of GLP-1, in the ileum and colon was increased in the OSM-treated group compared with the vehicle-treated group (Fig. 6e). In addition, the active GLP-1 levels in the ileum and colon were also upregulated in the OSM-treated group (Fig. 6f).

\section{Discussion}

Previously, we reported that deficiency of OSMR $\beta$ exacerbates adipose tissue inflammation, insulin resistance and hepatic steatosis under HFD conditions [15]. From the association of chronic inflammation in the adipose tissue with the development of insulin resistance [1-3], it can be expected that anti-inflammatory agents, such as salicylate, would be therapeutic candidates for the treatment of insulin resistance [17-19]. Thus, we hypothesised that OSM may exert antiinflammatory effects on adipose tissue inflammation, possibly leading to the improvement of metabolic disorders during obesity. In the present study, treatment of mice with OSM induced the shift of cytokine production profiles from inflammatory (Tnf- $\alpha, I l-1 \beta$ and Ifn- $\gamma$ ) to anti-inflammatory (Il-10 and $\mathrm{Il}-13$ ) in the adipose tissue with the phenotypic change of ATMs from M1 type to M2 type. OSM treatment decreased the serum concentrations of TNF- $\alpha$ and SAA, whereas the serum IL-10 concentration was increased. These results suggest that OSM suppresses systemic inflammation as well as adipose tissue inflammation partly through the antiinflammatory effects on ATMs.

In addition to adipose tissue inflammation, hepatic steatosis was also improved in the obese mice by the treatment with OSM. Previously, we reported that the expression of OSMR $\beta$ is increased in the liver of obese mice [15]. In the present study, intraportal administration of OSM revealed that OSM acted on the hepatocytes, increased the gene expression levels of Acsl3 and Acsl5 and reduced the expression of Fas in the liver of DIO mice. A previous study has reported that OSM induces the expression of ACSL3 and ACSL5 in HepG2 cells, a hepatocyte cell line [20]. These findings suggest that OSM has a direct effect on the liver, leading to the improvement of hepatic steatosis and insulin resistance. Such combined effects of OSM on the ATMs and liver may dramatically ameliorate the abnormal conditions of glucose and lipid metabolism in obese mice.

Energy balance is an equilibrium between energy intake, including food intake and nutrient absorption, and energy expenditure. In the present study, OSM decreased the lipid content in adipose tissue and liver as well as in serum, suggesting that it decreased the systemic lipid levels in the obese mice. The pair-feeding study revealed that the reduction in food intake partly contributed to these therapeutic effects of OSM on systemic lipid levels. In the hypothalamus, which is an important brain region for the regulation of food intake, OSMR $\beta$ was not detected. Instead, we observed that OSM increased the expression of active GLP-1 in the intestine of DIO mice. As GLP-1 decreases food intake via the vagus nerve in rodents [21, 22], OSM may reduce food intake through the upregulation of GLP-1 expression in the intestine.

On the other hand, the hypophagic effects of OSM did not wholly explain the cause of systemic lipid loss, as serum lipid levels were not significantly reduced by pair-feeding. In the present study, OSM increased the content of total lipid and triacylglycerol in the faeces in DIO mice, although the total quantity of faeces produced was not changed by OSM. In addition, the percentage of lipid absorption in the intestine was decreased by the treatment with OSM. However, OSM 
a

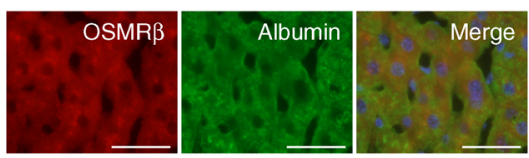

b
C

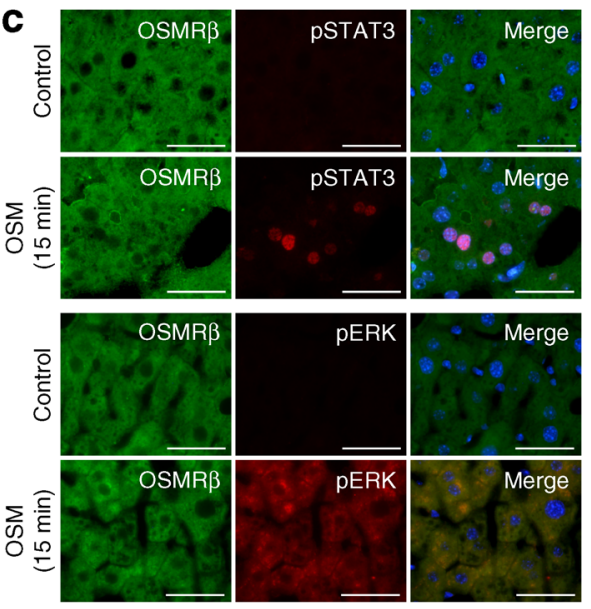

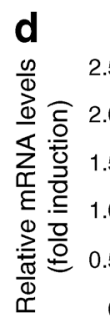
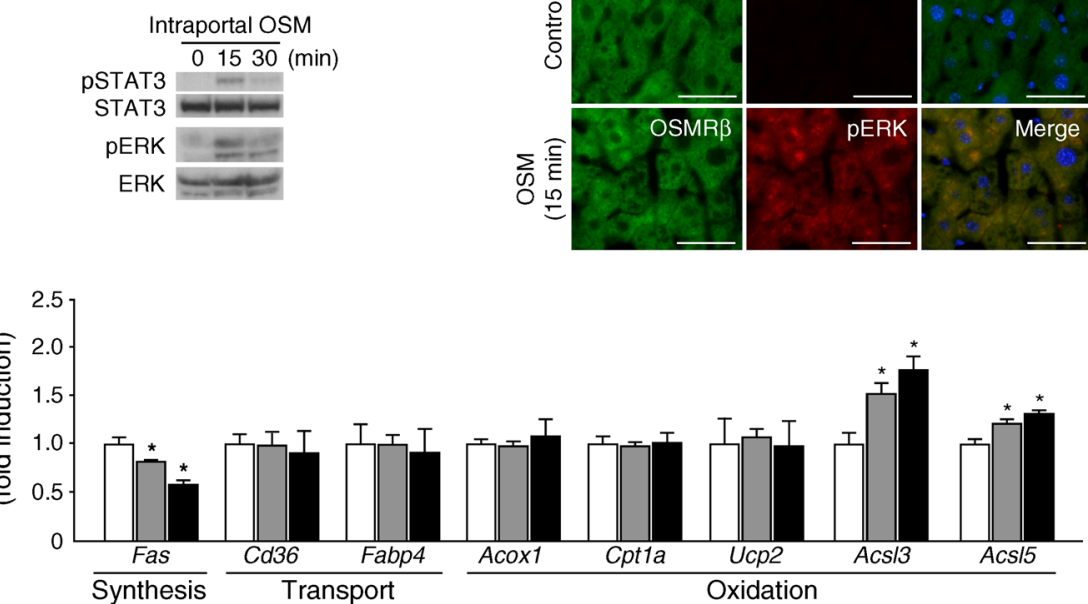

Fig. 5 The direct effects of OSM on lipid metabolism in the liver of DIO mice. DIO mice were injected intraportally with OSM (12.5 ng/g BW). (a) Double-immunofluorescence staining for OSMR $\beta$ (red) with albumin (green), a marker of hepatocytes, in the liver $(n=4)$. Nuclei were counterstained with DAPI (blue). Scale bar, $50 \mu \mathrm{m}$. (b) The phosphorylation of STAT3 and ERK in the liver $(n=4)$. (c) Double-immunofluorescence

affected neither energy expenditure nor serum ketone body levels. These findings suggested that OSM reduced body fat content by decreasing the lipid intake, including food intake and lipid absorption, rather than the expenditure of lipids.

a

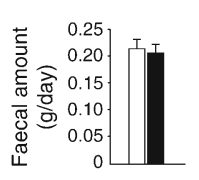

b

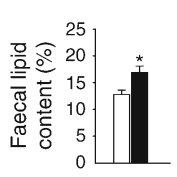

e

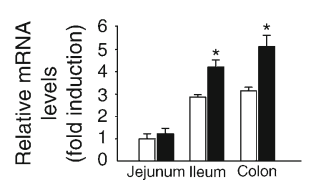

C

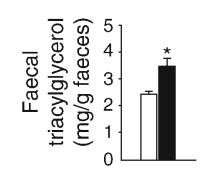

f

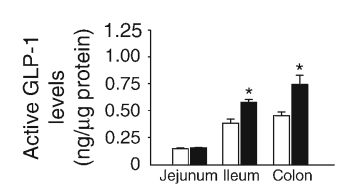

Fig. 6 Effect of OSM on lipid absorption and GLP-1 expression in the intestine of DIO mice. DIO mice were treated with OSM (12.5 ng/g BW) twice a day for 1 week. (a-d) The effect of OSM on lipid absorption was examined in DIO mice by measuring the total quantity of faeces produced (a), the percentage of total lipids in the faeces (b), the triacylglycerol content in the faeces (c) and the percentage of lipid absorption (d) in vehicle-treated and OSM-treated mice $(n=4)$. (e, f) The expression of proglucagon gene (e) and active GLP-1 levels (f) in the intestine of vehicle-treated and OSM-treated groups $(n=4)$. White bars, Vehicle; black bars, OSM. Data are means \pm SEM. ${ }^{*} p<0.05$ compared with Vehicle

staining for OSMR $\beta$ (green) with pSTAT3 (red) and pERK (red) in the liver $(n=4)$. Nuclei were counterstained with DAPI (blue). Scale bar, $50 \mu \mathrm{m}$. (d) The expression of genes related to fatty acid synthesis, fatty acid transport and fatty acid oxidation in the liver $(n=4)$. White bars, Control; grey bars, OSM $1 \mathrm{~h}$; black bars, OSM $2 \mathrm{~h}$. Data are means \pm SEM. ${ }^{*} p<0.05$ compared with Control

Current therapeutic approaches to obesity-related metabolic diseases focus on adiponectin and fibroblast growth factor 21 (FGF21), which are known to have beneficial effects on the underlying insulin resistance in obese mice [23, 24]. However, although treatment with adiponectin at a dose of $1.5 \mathrm{mg}(\mathrm{kg}$ $\mathrm{BW})^{-1} \mathrm{day}^{-1}$ for 2 weeks alleviates insulin resistance and hepatic steatosis, adiponectin cannot reduce the BW of obese mice at this dose [25]. The treatment of obese mice with FGF21 at a dose of more than $0.5 \mathrm{mg}(\mathrm{kg} \mathrm{BW})^{-1}$ day $^{-1}$ decreases the BW and ameliorates insulin resistance and hepatic steatosis [26]. In contrast, just 1 week of treatment with OSM $\left(25 \mu \mathrm{g}[\mathrm{kg} \mathrm{BW}]^{-1} \mathrm{day}^{-1}\right)$ improved metabolic disorders, including obesity, insulin resistance and hepatic steatosis, in the obese mice. These findings suggest that OSM can exert its effects on obesity-related metabolic disorders with a shorter dosing period and smaller dose than FGF21 and adiponectin.

Furthermore, it has been reported that DIO mice are in an FGF21-resistant state [26]. The expression of OSMR $\beta$ is increased in the adipose tissue and liver of obese mice [15], suggesting that the sensitivity to OSM in such organs is elevated. Therefore, OSM seems to be a candidate for a powerful therapeutic agent without unfavourable side effects in the treatment of obesity-related metabolic diseases. Recently, it has also been reported that the metabolic effects of FGF21 are mediated by adiponectin (the so-called 'FGF21- 
adiponectin axis') [27, 28]. In the present study, treatment with OSM did not induce an increase in serum adiponectin levels, suggesting that OSM exerts its metabolic effects independent of adiponectin. Thus, combined therapy with OSM and adiponectin or FGF21 may be more powerful in the treatment of obesity-related metabolic diseases.

In conclusion, treatment with OSM dramatically improved obesity, adipose tissue inflammation, insulin resistance and hepatic steatosis in both DIO and $o b / o b$ mice. These results suggest that OSM is a promising therapeutic candidate for the treatment of obesity-related metabolic diseases.

Funding This work was supported by a Grant-in-Aid for Scientific Research (B) (JSPS KAKENHI Grant Number 26282195) from the Japan Society for the Promotion of Science and Research Grant on Priority Areas from Wakayama Medical University.

Contribution statement TK and YM contributed to the conception and design of this study. The acquisition of data was carried out by TK and the analysis of data was carried by TK, MT, HF and YM. All authors contributed to the interpretation of data, participated in drafting the article and revising it critically for important intellectual content, and approved the final version to be published. YM is responsible for the integrity of the work as a whole.

Duality of interest The authors declare that there is no duality of interest associated with this manuscript.

\section{References}

1. Xu H, Barnes GT, Yang Q et al (2003) Chronic inflammation in fat plays a crucial role in the development of obesity-related insulin resistance. J Clin Invest 112:1821-1830

2. Patsouris D, Li P-P, Thapar D, Chapman J, Olefsky JM, Neels JG (2008) Ablation of CD11c-positive cells normalizes insulin sensitivity in obese insulin resistant animals. Cell Metab 8:301-309

3. Osborn O, Olefsky JM (2012) The cellular and signaling networks linking the immune system and metabolism in disease. Nat Med 18: 363-374

4. Després J-P, Lemieux I (2006) Abdominal obesity and metabolic syndrome. Nature 444:881-887

5. van Herpen NA, Schrauwen-Hinderling VB (2008) Lipid accumulation in non-adipose tissue and lipotoxicity. Physiol Behav 94:231-241

6. Sun B, Karin M (2012) Obesity, inflammation, and liver cancer. J Hepatol 56:704-713

7. Park EJ, Lee JH, Yu G-Y et al (2010) Dietary and genetic obesity promote liver inflammation and tumorigenesis by enhancing IL-6 and TNF expression. Cell 140:197-208

8. Tanaka M, Miyajima A (2003) Oncostatin M, a multifunctional cytokine. Rev Physiol Biochem Pharmacol 149:39-52

9. Lindberg RA, Juan TSC, Welcher AA et al (1998) Cloning and characterization of a specific receptor for mouse oncostatin M. Mol Cell Biol 18:3357-3367

10. Tanaka M, Hara T, Copeland NG, Gilbert DJ, Jenkins NA, Miyajima A (1999) Reconstitution of the functional mouse oncostatin M (OSM) receptor: molecular cloning of the mouse OSM receptor $\beta$ subunit. Blood 93:804-815

11. Tamura S, Morikawa Y, Miyajima A, Senba E (2002) Expression of oncostatin M in hematopoietic organs. Dev Dyn 225:327-331

12. Repovic P, Benveniste EN (2002) Prostaglandin E2 is a novel inducer of oncostatin-M expression in macrophages and microglia. J Neurosci 22:5334-5343

13. Goren I, Kämpfer H, Müller E, Schiefelbein D, Pfeilschifter J, Frank S (2006) Oncostatin M expression is functionally connected to neutrophils in the early inflammatory phase of skin repair: implications for normal and diabetes-impaired wounds. J Invest Dermatol 126:628-637

14. Komori T, Tanaka M, Senba E, Miyajima A, Morikawa Y (2013) Lack of oncostatin $\mathrm{M}$ receptor $\beta$ leads to adipose tissue inflammation and insulin resistance by switching macrophage phenotype. J Biol Chem 288:21861-21875

15. Komori T, Tanaka M, Senba E, Miyajima A, Morikawa Y (2014) Deficiency of oncostatin M receptor $\beta$ (OSMR $\beta$ ) exacerbates highfat diet-induced obesity and related metabolic disorders in mice. J Biol Chem 289:13821-13837

16. Kang HW, Niepel MW, Han S et al (2012) Thioesterase superfamily member $2 /$ acyl-CoA thioesterase 13 (Them2/Acot13) regulates hepatic lipid and glucose metabolism. FASEB J 26:2209-2221

17. Yuan M, Konstantopoulos N, Lee J et al (2001) Reversal of obesityand diet-induced insulin resistance with salicylates or targeted disruption of IKK $\beta$. Science 293:1673-1677

18. Donath MY, Shoelson SE (2011) Type 2 diabetes as an inflammatory disease. Nat Rev Immunol 11:98-107

19. Goldfine AB, Fonseca V, Jablonski KA et al (2013) Salicylate (Salsalate) in patients with type 2 diabetes: a randomized trial. Ann Intern Med 159:1-12

20. Zhou Y, Abidi P, Kim A et al (2007) Transcriptional activation of hepatic ACSL3 and ACSL5 by oncostatin M reduces hypertriglyceridemia through enhanced $\beta$-oxidation. Arterioscler Thromb Vasc Biol 27:2198-2205

21. Abbott CR, Monteiro M, Small CJ et al (2005) The inhibitory effects of peripheral administration of peptide $\mathrm{YY}_{3-36}$ and glucagon-like peptide-1 on food intake are attenuated by ablation of the vagal-brainstem-hypothalamic pathway. Brain Res 1044: $127-131$

22. Talsania T, Anini Y, Siu S et al (2005) Peripheral exendin-4 and peptide YY3-36 synergistically reduce food intake through different mechanisms in mice. Endocrinology 146:3748-3756

23. Yamauchi T, Kamon J, Waki H et al (2001) The fat-derived hormone adiponectin reverses insulin resistance associated with both lipoatrophy and obesity. Nat Med 7:941-946

24. Xu J, Lloyd DJ, Hale C et al (2009) Fibroblast growth factor 21 reverses hepatic steatosis, increases energy expenditure, and improves insulin sensitivity in diet-induced obese mice. Diabetes 58:250-259

25. Xu A, Wang Y, Keshaw H, Xu LY, Lam KSL, Cooper GJS (2003) The fat-derived hormone adiponectin alleviates alcoholic and nonalcoholic fatty liver diseases in mice. J Clin Invest 112:91-100

26. Fisher FM, Chui PC, Antonellis PJ et al (2010) Obesity is a fibroblast growth factor 21 (FGF21)-resistant state. Diabetes 59:27812789

27. Lin Z, Tian H, Lam KSL et al (2013) Adiponectin mediates the metabolic effects of FGF21 on glucose homeostasis and insulin sensitivity in mice. Cell Metab 17:779-789

28. Holland WL, Adams AC, Brozinick JT et al (2013) An FGF21adiponectin-ceramide axis controls energy expenditure and insulin action in mice. Cell Metab 17:790-797 